\title{
The Asymmetry of Chinese/English Action-Result Events Encoding: A Cognitive Approach
}

\author{
Duxin Cao \\ College of International Studies, Southwest University, Chongqing, China \\ Email: 751844769@qq.com
}

Received March $14^{\text {th }}$, 2013; revised April 15 ${ }^{\text {th }}$, 2013; accepted April 21 $1^{\text {st }}, 2013$

\begin{abstract}
Copyright ( 2013 Duxin Cao. This is an open access article distributed under the Creative Commons Attribution License, which permits unrestricted use, distribution, and reproduction in any medium, provided the original work is properly cited.
\end{abstract}

\begin{abstract}
The present paper attempts to look at Chinese resultative verb compounds form the perspective of cognitive relativism, namely through the contrastive study of Chinese and English verb-resultatives. Based on the comparison, the present thesis tries to explore the underlying conceptual approaches to structuring events consisting of both action and result by Chinese native speakers and English native speakers and show the discrepancies between them in cognition or more specifically, conceptualization of reality. Resultative verb compounds in Chinese are analyzed in terms of Talmy's conceptual structure and are shown to present a problem for Talmy's well-known typological dichotomy between "verb-framed" and "satellite-framed" languages. It is also argued that the so-called "resultative complement" in Chinese resultative verb compounds can be treated as the center of predication, even as the main verb. Pending further psycholinguistic evidence, it appears that Chinese speakers place relatively more emphasis on the result of an event, whereas English speakers more on the process of an event.
\end{abstract}

Keywords: Cognitive Approach; Relativism; Conceptual Structure; Chinese and English Verb-Resultatives

\section{Introduction}

\section{Research Background}

Resultative constructions have been a hotly-debated topic in Chinese linguistics. This chapter gives an overview of resultative constructions and their classifications and the research scope of this essay is thus defined.

A resultative verb compound usually encodes a causing event (a process, or an activity) and a caused event (a result state). Two related constructions exist in Mandarine Chinese, which are not mutually reducible: the resultative verb compounds (hereafter RVCs, namely, a compound verb made up of two parts, the first indicating an action and the second the result of that action) as in (1a) and the resultative-de constructions as in (1b):

(1a) Ta pao lei le. He/She run-tired ASP (ASP stands for aspect marker) "He/She ran him/herself tired."

(1b) Ta pao de hen lei. He/She run RES very tired (RES stands for resultative-de)

"He/She ran him/herself very tired."

However, RVCs attract more attention than resultative-de constructions, owing to their diverse behaviours in terms of argument linking, potential interpretations, and degree of analyticity and productivity. Thus we will follow this trend and focus on RVCs in this paper.

Different from previous approaches, in this paper the writer attempts to look at Chinese resultative verb compounds form the perspective of cognitive relativism, namely through the contrastive study of Chinese and English verb-resultatives and solve the problems of the previous studies as well. Based on the comparison, the present thesis tries to explore the underlying conceptual approaches to structuring events consisting of both action and result by Chinese native speakers and English native speakers and show the discrepancies between them in cognition or more specifically, conceptualization of reality. In the spirit of linguistic relativism, the author would like to view the difference as reflecting ontological relativity involving events. This ontological relativity means that English speakers tend to attend relatively more to the process of an event, but, in contrast, Chinese relatively more to the result. In other words, while English is an agent-oriented language, Chinese is a patient-oriented language. The other goal of this paper is to argue against the dominant view that V1 is the head of RVCs and to explicate that $\mathrm{V} 2$ is the center of predication of the action-result verb compounds, and therefore, can be treated as the main verb. If we take V2 as the main verb, then Chinese is no longer a satelliteframed language as Talmy has claimed. Instead, it makes more sense to view Chinese as primarily a verb-framed language and only secondarily a satellite-framed language, thus forming a tentative amendment to Talmy's well-known typological dichotomy between "verb-framed” and "satellite-framed” languages.

This paper proceeds in the following order. Chapter one opens with the significance and purpose of this studies, then proceeds to a review made on previous researches done in the field of RVCs. The deficiency of the previous researches is also 
pointed out and analyzed in this chapter. In Chapter 2, the current author establishes the theoretical framework of the intended cognitive relativism model for the contrastive studies of Chinese and English verb-resultatives. Chapter 3 presents the semantic category "result" in Chinese and its differences from that in English. Chapter 4 is to testify the claim that in Chinese V2 is the center of predication of the action-result verb compounds, and therefore, can be treated as the main verb by analyzing some concrete language materials. Chapter 5 ends the thesis by summarizing the major findings of the study, pointing out its theoretical and practical implications and also limitations and proposing suggestions for further research in this area.

Next is a general picture of what has been accomplished in the study of Chinese verb-resultatives.

\section{Literature Review}

There are lots of previous works on Mandarin resultatives which we cannot go through in the present paper. There are approaches as diverse as lexical, complex predicate, verb class, and aspectual ones, among others. It is our aim to have a deeper understanding of them before we proceed to our own analysis.

As regards lexical approach, Thompson (1973) discusses how Mandarin RVCs are formed by lexical rules; Simpson (1983) proposes a lexical rule to account for the valencychanging property of English resultatives; Li (1999) shows how argument structures of the two component verbs and that of the RVCs are related.

On the assumptions that unaccusative verbs are underlying objects and that the fake reflexives are coreferential with the subjects, Simpson (1983: p. 148) shows that "resultative attributes in English are subject to the SYNTACTIC constraint that they must be controlled by an OBJECT, whether underlying or surface”. This constraint is later dubbed the Direct Object Restriction (DOR) in Levin and Rappaport Hovav.

Li's (1999) pivotal work on V-V compounds argues that the argument structure of the compound is determined compositionally from that of each component verb. Under the Generative Grammar framework he proposes the following requirements: 1) Theta-identification; 2) Structured theta-grid; 3) Headfeature percolation.

Under Lexical Functional Grammar, Her (2007) gives a lexical mapping account of Mandarin resultatives. The framework assumes an a-structure (argument structure) which mediates between the lexical semantic structure and the syntactic structure of a predicator.

The lexical approach of resultatives either employs lexical rules (Thompson, 1973), or augments verbal arguments (Simpson, 1983), or projects argument structures from those of component verbs ( $\mathrm{Li}, 1999$; Her, 2007). It either disregards Type object-oriented resultatives or explains these resultatives in a controversial way. None of them cover resultatives of objectoriented transitive resultatives, which have nonsubcategorized objects. Thus the lexical approach is not tenable in accounting for Mandarin RVC constructions.

Complex predicate approach presents a view that the result part and the main verb in a resultative construction form a constituent, or a complex predicate, which licenses the grammatical object (if any) and the grammatical subject. Representative of this approach is Huang's (1992) proposal that RVCs must be handled on a par with resultative-de constructions and that the result verb (or result phrase in the case of resultative-de) form a complex predicate with the main verb. Rapoport's (1993) paper discusses four constructions: causatives, resultatives, argument small clauses, and adjunct-predicate constructions, the former three being analyzed as containing complex predicates.

Huang's (1992) account of the causative-inchoative alternations of RVCs and resultative de-constructions is appealing in terms of simplicity. It also has an important virtue of recognizing that "theta-roles are compositionally assigned" (Huang, 1992: p. 130). That is, the verb and the result form a complex predicate, which licenses the grammatical object and the grammatical subject. This analysis, however, fails to explain some cases where an added external argument is not allowed.

Rapoport (1993) argues that the verb and the result form a complex predicate in English resultative constructions. He also concludes that resultative constructions cannot be analyzed as projections of the verbs. Instead, both the verb and the result contribute to the argument realization of grammatical functions. Therefore, the complex predicate approach excels the lexical approach in that the licenser is not limited to the verb only, but to a larger language construct.

However, the complex predicate analysis still fails to explain the existence of non-subcategorized objects in resultative constructions, object oriented resultatives in Mandarin.

With regard to verb class approach, Cheng and Huang (1994) correlate event types with verb classes (unaccusativity of verbs) and discuss the possibility of causativization. This influential work raised some significant issues in Mandarin resultatives that concerned us in this thesis. Cheng and Huang (1994: p. 188) demonstrate the four basic predicate types: unergative, transitive, ergative, and causative. Likewise, they argue that Chinese RVCs can be classified accordingly. From the point of view of verb classes, they argue that "the argument structure of a compound is essentially a composition of the event structure rather than the transitivity properties, of its component parts...” (p. 187). They claim that there are two paradigms: the active paradigm performs the unergative/transitive alternation, while the non-active paradigm performs the ergative/causative alternation. The unergative/transitive alternation involves the event type "activity", while the ergative/causative alternation involves the event type "(change of) state” (pp. 188-189).

The deficiency of Cheng and Huang's (1994) analysis comes from ignoring the differences between object-oriented RVCs and subject-oriented RVCs in transitive sentences, only the latter qualified for unergative-transitive alternations (since the grammatical subjects remain the "semantic hosts" of the result in both unergative and transitive constructions). Thus the fourway distinction above is not appropriate for

Mandarin resultatives. We argue that the complexity results from the complexity in Vc's and Vr's event structures, both contributing to the behavior of the RVCs. The puzzling nature of the unergative verbs (having both transitive and causative alternations) is due to that they can be regarded as "unergative-unaccusative” complexes, the first giving rise to transitive alternation and the second causative alternation.

The following part presents the aspectual approach of resultative constructions. Both Rappaport Hovav and Levin (2001) and J. Chang (2003) employ the concept of a causal chain, though they differ in some details.

Rappaport Hovav and Levin (2001) propose a semantic account of English resultatives from an event structure viewpoint and provide an argument against the DOR (Direct Object Restriction). They argue that the difference in surface form re- 
flects the difference in event structure and further argue that the grammaticality of resultatives lies in the well-formedness of event structures represented by causal chains.

J. Chang (2003: p. 317) argues that "it is the event role an argument plays in event structure, rather than the thematic role an argument plays, that determines how and where the argument is linked to the syntax." He proposes three event roles (i.e. event participants) involved in a causal chain (J. Chang, 2003: p. 330): The Initiator is "an entity that is involved in the initiation or bringing about of an object." The Target of activity is "an entity that undergoes an action." The Locus of affect is "an entity that is involved in the endpoint or resulting state.” These event participants are linked to syntactic positions via linking rules.

Rappaport Hovav and Levin's (2001) work faces at least two challenges if the same framework is applied to Mandarin. The first challenge is the plausibility of the Argument-per-Subevent condition, which explains the difference between the bare XP pattern and the reflexive pattern of English. The second challenge is the prediction of the causal chains, which predict that object-oriented readings (nonbranching causal chains) are accepted while subject-oriented readings (branching causal chains) must be excluded, a violation of the fact that in Mandarin subject-oriented resultatives exist, if not so common.

J. Chang's (2003) analysis is promising in incorporating Verb-copying construction and $\mathrm{Ba}$-construction. The isomorphism between the temporal order of event roles and the linear order of syntactic constituents is also appealing. However, there are issues left unmentioned at all in his work. First, the inverted causative resultatives are not discussed. Second, the subjectoriented resultatives of the form [NP1 Vc-Vr NP2] are exemplified by only one example [chi-bao fan] "eat-full rice"; nothing is said about the restriction of their occurrence and their relation to other resultatives.

To summarize, the aspectual approach is superior to traditional approaches in recognizing the existence of nonsubcategorized objects. The causal chain functions like a "licenser" of these objects. As we have pointed out here, however, there is still weakness in this approach.

In this chapter, we have introduced different approaches to resultative constructions in English and Mandarin. The lexical approach is a bottom-up approach which is theoretically plausible, but it cannot generate resultatives with nonsubcategorized objects. The complex predicate approach recognizes the contribution of both component verbs in argument licensing, but it does not deal properly with the nature of causation in Mandarin resultatives. The verb class approach is insightful in its fourway distinction, but it cannot explain alternations in Mandarin resultatives property. The aspectual approach fails to cover different types of resultatives. Thus we see that there are pros and cons for each approach.

To draw on their strength and avoid their weakness, in this paper the writer attempts to look at Chinese resultative verb compounds form the perspective of cognitive relativism, namely through the contrastive study of Chinese and English verbresultatives and solve the problems of the previous studies as well. Based on the comparison, the present thesis ties to explore the underlying conceptual approaches to structuring events consisting of both action and result by Chinese native speakers and English native speakers and show the discrepancies between them in cognition or more specifically, conceptualization of reality. We will introduce the cognitive approach in the next chapter.

\section{Theoretical Framework}

\section{Cognitive Linguistics and Linguistic Relativity}

The study of conceptualization of reality in different languages and cultures has been enthusiastically pursued by anthropologists and psychologists, especially in the well-known Boas-Sapir-Whorf tradition. In contrast, American structuralists and generative grammarians have shunned away from the study of language as capable of reflecting conceptualization in different cultures. In retrospect, we can perhaps identify three main reasons for linguists to have taken a very different approach to the study of language. First, psycholinguistic experiments have generally failed to confirm either strong or weak versions of the Sapir-Whorf hypothesis, that is, linguistic determinism or linguistic relativity. For instance, in reference to Chinese grammar, Bloom's (1981) controversial hypothesis regarding the absence of overt counterfactual grammatical devices in Chinese and its effect on the thought of native speakers of Chinese have been repeatedly challenged (cf. Wu, 1994). Second, American structuralists and generative grammarians have subscribed to Saussure's arbitrariness principle of linguistic signs and believed in the autonomy of syntax. The third reason has to do with the influential view shared by philosophers (e.g., Fodor), linguists (e.g., Chomsky) and cognitive scientists (e.g., Pinker) that language is independent of culture and thought and that the mental representation of language involves only symbols and their operations but not images.

The emergence of cognitive linguistics as developed by Lakoff, Langacker, and Talmy in the last three decades can be viewed as a revival of the interest in the study of conceptualization of reality by language in different cultures. In his very recent work, Talmy (2000: pp. 1-5) has characterized cognitive linguistics as a conceptual approach to the study of language, in contrast with the formal approach adopted in the tradition of generative grammar and the psychological approach as practiced by cognitive psychologists. Furthermore, as pointed by Talmy (ibid.), cognitive linguistics also addresses the concerns of the two other approaches to language, for cognitive linguistics seeks to understand the formal structure of language as patterns of organization of conceptual content in language from the perspective of general cognitive mechanism (Wang, 2008). In fact, this is also the endeavor of the Boas-Sapir-Whorf tradition, although it places emphasis on cognitive relativity as well as cognitive universality. Therefore, the emergence of cognitive linguistics calls for a new interest in cognitive relativism.

Lakoff in his famous book Women, Fire, and Dangerous Things (1987: pp. 304-337) has devoted a whole chapter to Whorf and relativism. In contrast, Langacker $(1987,1991)$ has not explicitly addressed the issue of linguistic relativity in his seminal works on the foundations of cognitive linguistics. However, the attempt to restate the Boasian conceptual approach to language seems to be very clear in Langacker's view of language structure. Thus, according to Langacker, "if one language says I am cold, a second I have cold, and a third It is cold to me, these expressions differ semantically even though they refer to the same experience, for they employ different images to structure the same basic conceptual content" (1987: p. 47). He thus claimed (ibid.) that "meaning is language-specific to a considerable extent" and that "full universality of semantic structure 
cannot be presumed even on the assumption that human cognitive ability and experience are quite comparable across cultures.” In short, it appears that the impact of cognitive linguistics can be made stronger in the context of linguistic relativism and that cognitive linguistics can serve as a modern approach to linguistic relativity.

\section{Relatvism and Construction of Chinese Grammar}

As pointed out by Lakoff (1987: pp. 306-337), there are many different views of what relativism is. For the present purpose, the writer shall not attempt to define his own version in answering to the host of questions which Lakoff has put for distinguishing different varieties of relativism. Instead, the author shall take a position that is no different from the original Boasian approach which aims to describe the grammars of non-Indo-European languages in their own terms rather than in terms of the meta-language developed from the structure of Indo-European languages, which is also actively advocated at home by Shen Jiaxuan (2011).

The Boasian approach should be greatly appreciated in the analysis of Chinese grammar. For one reason, there is no indigenous Chinese grammar. The only two indigenous Chinese grammatical concepts are "full words" and "empty words" developed from the study of classical Chinese. For another reason, research on Chinese grammar since Mashi Wentong in 1898 has invariably been based on grammatical theories derived from studies of Indo-European languages. Chinese grammarians have relied heavily on English translations and on grammatical theories of English to analyze Chinese. It is not at all surprising that the result of the objectivist approach based on truth-conditional semantics supports the main theme of generative grammar that languages are largely no different from each other in structuring principles. There is perhaps nothing wrong with using translation as a heuristic device to analyze Chinese or any non-Indo-European language that does not have its own indigenous grammar. However, this kind of objective approach assumes not only that semantics is universal, but also that structural relationships among sentences are also universal. This assumption makes the search for linguistic universals easier. But on closer observation, it is superficial at best, and fallacious at worst. The fact is that Chinese sentence patterns are structured with each other under a set of conceptual systems on the one hand, and, on the other, that English sentence patterns are also structured with each other under another set of conceptual systems. An instructive example of misconception due to the objectivist approach in research on Chinese grammar during the last century can be found in the analysis of active and passive sentences in Chinese. Thus, with rare exception, Chinese grammarians, be they structuralist or generativist, have invariably treated (2a) as the active construction and (2c) as its corresponding passive. Syntacticians who have worked on language typology and language universals have also taken this analysis for granted. However, as pointed out in Tai (1989), on both semantic and syntactic grounds, (2b) and not (2a) should be treated as the corresponding active for the passive sentence (2c).

(2) a. Ta mai-le chezi. he sell-ASP car

"He sold the car."

b. Ta ba chezi mai-le.

"He sold the car."

\author{
c. Chezi bei ta mai-le. \\ "The car was sold by him.”
}

In fact, the conceptual approach with a tint of relativism to the study of Chinese grammar has uncovered several important conceptual principles underlying the organization of Chinese grammar. They include the Principle of Temporal Sequence (Shen, 1993), the Principle of Whole-and-part (Tai 1989), certain iconic constraints on the denominal verb convention in Chinese and iconic motivations for verb-copying in Chinese (Tai, 1999). The author believes that within the framework of the conceptual approach, many more conceptual principles of great explanatory value are to be uncovered. In the following section, the writer shall show that the semantic category "result" is a semantic prime in Chinese verb semantics and the action-result schema has played a much more important role in the Chinese conceptual system than in English.

\section{The Discrepancies of Semantic Category "Result" in Chinese and English}

In Talmy's (2000, Chapters 1\&2) framework of cognitive semantics, "result" is a semantic category under co-event which accompanies the main event's action or state. Although the category of "result" is expressed in both Chinese and English, it has different ranges of meaning which provide motivations for different syntactic patterns.

Consider the following contrast between Chinese and English sentences.

(3a) Ta jia-cuo-le laogong. she marry-wrong-ASP husband "She has married the wrong husband."

(3b) Ta qu-cuo-le laopo. he marry-wrong-ASP wife "He has married the wrong wife."

(4) Ta zou-jin-le gongyuan. s/he walk-enter-ASP park "S/he walked into the park."

(5) Ta ku-hong-le yanjing s/he cry-red-ASP eye "S/he cried so hard that her/his eyes turned red."

(6) Women yao wu-chu jiankang. we want dance-out health "We want to dance to become healthy."

(7) (Tamen chi yao) chi-chu wenti. they eat medicine eat-out problem "They became unhealthy from taking medicine."

(8) Ta jintian zhi pao-dao-le san-ge keren. he today only run-reach-ASP three-CL customer "He (taxi driver) has only run three trips today."

The sentences in (3a) and (3b) illustrate a systematic difference between Chinese and English in describing situations wherein a mistake occurred. While the Chinese word cuo "wrong" is the resultative component in action-result verb compounds indicating the result of an action, the English word "wrong" is an adjective modifying the object noun. If one takes an objectivist approach and assumes Chinese and English have the same semantics describing the making of mistakes, one would perhaps be inclined to subscribe to the principle and parameter approach to account for the difference between the two languages. On the other hand, if we take a non-objectivist 
approach, we immediately see the difference in (3a) and (3b) as the grammatical embodiment of two different conceptual systems that are equally effective. Chinese speakers attribute the mistake as a result of the action that the subject performs. In contrast, English speakers report a discrepancy between the person s/he sets out to marry and the person s/he has actually married. Similarly, the action-result schema is consistently patterned in Chinese, as shown in sentences (4) to (8). In contrast, the corresponding English sentences are largely expressed with different grammatical patterns in which the result is usually implied, rather than overtly expressed as in Chinese.

It is clear from the above examples that the action-result schema provides a unified conceptual schema for describing various situations which are not necessarily construed as action-result schema in English, even though English does have an action-result schema as illustrated below.

(9) He hammered the metal flat.

(10) He kicked the door open.

(11) He painted the house red.

The recognition of "result" as a semantic prime in Chinese verb semantics in fact forms a contrast with the four semantic categories which Vendler (1967) has proposed for English (namely, state, activities, accomplishment and achievement categories), for Chinese has only state, activities, and result, lacking accomplishment and achievement categories. The latter two categories are expressed mostly in action-result verb compounds (V1-V2). Moreover, the resultative complement V2 seems to indicate foreground information and the action verb V1 seems to indicate background information.

The followings are some key arguments regarding to these cross-linguistic discrepancies. First, while accomplishment verbs in English necessarily imply an attainment of the goal, their seeming equivalents in Chinese do not necessarily imply so. For instance, the accomplishment verb "to kill” in English necessarily implies the death of the recipient of the action. Therefore, (12) is ungrammatical in English.

(12) *I killed John, but he didn’t die.

The verb sha in Chinese is assumed in most English-Chinese and Chinese-English dictionaries as equivalent to "to kill" in English. However, as shown in (13), the verb sha doesn't necessarily imply the death of the recipient of the action (If context information is properly provided, the verb sha can carry a pragmatic connotation implying the death of the recipient of the action. When the verb sha is used in bei and ba constructions, the implication of death tends to be stronger. It is also the case in verb compound mousha "murder.").

(13) Wo sha-le John liang-ci, ta dou mei si. I kill-ASP John two-CL he all not die "I performed the action of attempting to kill John twice, but he didn't die.”

To guarantee the death of the recipient of the action, the verb compound sha-si has to be used. The ungrammaticality of (14) shows that sha-si does imply the death of the recipient of the action.

(14) *Wo sha-si-le John liang-ci, ta dou mei si. I kill-die-ASP John two-CL he all not die *“I killed John twice, but he didn’t die.”
In fact, the current author would argue that the verb "to kill" doesn't really exist in Chinese. On the one hand, many Chinese action-result verb compounds involving si "to die" can be translated into "to kill” in English. For example,

(15) kache nian-si-le John. truck grind-die-ASP John “The truck killed John by running him over."

(16) ta qiao-si-le John. he knock-die-ASP John

"He killed John by hitting him with a hammer (stone, stick, etc.)."

(17) ta da-si-le John. he hit-die-ASP John "He killed John by hitting him (with or without an instrument).”

(18) ta ba erzi e-si-le. he take son starve-die-ASP "He killed his son by starving him."

On the other hand, there are many uses of "to kill” in English that cannot be translated with sha-si. They need to be translated with verb compounds in construction with -si. For instance,

(19) The earthquake killed hundreds of people.

(20) The famine killed thousands of people.

(21) He was killed in an accident.

Second, among those achievement verbs identified by Vendler (1967) and Dowty (1979), many of them are expressed in Chinese by action-result verb compounds. For example, "to find" in Chinese is zhao-dao "seek-reach", "to receive” is shoudao "collect-reach," "to see” is kan-dao "look-reach," and "to hear" is ting-dao "listen-reach." These resultative verb compounds behave syntactically and semantically no different from those equivalent to accomplishment verbs in English mentioned above.

Most action-result verb compounds in Chinese function as transitive verbs. There have been two related issues regarding the transitivity. The first issue is to identify the "main verb" or "head" in these compounds. The second issue has to do with the source of transitivity. Here, the present paper shall be mainly concerned with the first issue. The dominant view holds that the first element of the compounds which represent action is "main verb" or "head" (Chao, 1968; Li \& Thompson, 1981; Huang, 1988; Chang, 2001). A different view has been proposed by Shen (1993) and Li (2011) which regards the second element, or the so-called "complement", as the "head.” If we accept "result" as a semantic prime underlying action-result verb compounds, it makes sense to take the second element as the center of predication, even though it cannot be analyzed as an independent transitive verb in surface syntax. Thus, the verb compounds in construction with si "to die" illustrated in sentences (15) through (18) can be analyzed as "cause to die", as is claimed by Wang Qiong and Wu Yaqing (2005). In other words, as second element of the compound, it is really equivalent to "to kill" in English. It functions as the center of predication, if not the main verb in surface syntax. The action verbs in these compounds, regardless of whether they are transitive or intransitive verbs, function like manner adverbs, which is shared by Liu and Zhang (2012). As a matter of fact, the first element in verb compounds with si doesn't have to be a verb by itself. For example, $d u$ in (22) and $q i$ in (24) cannot stand alone as a verb 
as illustrated in (23) and (25), respectively.

(22) Tamen du-si-le John. they poison-die-ASP John "They killed John with poison."

(23) *Tamen du-le John. they poison-ASP John "They poisoned John."

(24) Zhe-jian shi qi-si-le John. this-CL thing anger-die-ASP John "This case infuriated John to death."

(25) *Zhe-jian shi qi-le John. this-CL thing anger-ASP John

In fact, in Talmy's (2000: pp. 151-153) recent treatment of the semantic category "result", all incorporation of "result," whether in verb root or satellite, presents the main event. In Chinese action-result verb compounds (V1-V2), V1 expresses the cause, but presents the subordinate event; whereas V2 expresses the result, but presents the main event. Thus Talmy's analysis of the resultative construction supports the writer's analysis of the resultative complement as the center of predication in Chinese. However, Talmy treats the resultative complement as the satellite rather than verb root in Chinese. In the following section, the author questions the analysis and raises the issue whether Chinese is indeed a "satellite-framed" language like English.

\section{Resultative Complement as Verb Root in Chinese}

Talmy (1985, 2000) has proposed a conceptual analysis of motion events which consists of four cognitive components: FIGURE, GROUND, MOTION, and PATH. In addition to these four internal components, a motion event is accompanied with an external co-event which includes MANNER and CAUSE. In some languages such as English or German, the verb incorporates MOTION and MANNER. In other languages such as French or Spanish, the verb incorporates MOTION and PATH. The former languages are referred to as "satelliteframed" languages and the latter as "verb-framed" languages. In satellite-framed languages the cognitive component PATH has to be spelled out, while in verb-framed languages the cognitive component MANNER has to be spelled out. This contrast can be illustrated by the English example in (26) and the French example in (27) from Ungerer and Schmid (1996).

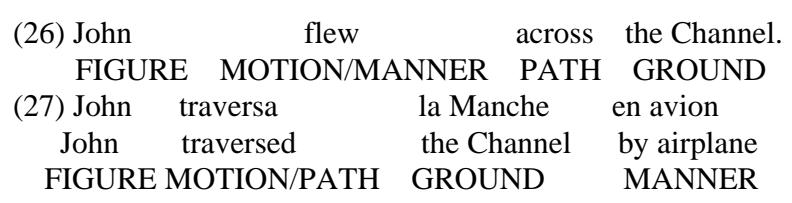

The following Spanish examples from Talmy (2000: pp. 4950) also illustrate the same point.
(28) La botella entró a la cueva (flotando) the bottle MOVED-in to the cave (floating) "The bottle floated into the cave."
(29) La botella salió de la cueva (flotando) the bottle MOVED-out from the cave (floating) "The bottle floated out of the cave."
(30) La botella pasó por la piedra (flotando) the bottle MOVED-by past the rock (floating) "The bottle floated past the rock."

Talmy has classified Chinese as a satellite-framed language because the verb in Chinese incorporates the MANNER component, but not the PATH component, as shown in (31).

$$
\begin{array}{ccl}
\text { (31) John } & \text { fei } & \text { guo Yingjili Haixia } \\
\text { John } & \text { fly } & \text { pass English Channel } \\
\text { FIGURE } & \text { MOTION/MANNER } & \text { PATH GROUND }
\end{array}
$$

However, the cognitive component PATH guo in (31) can be used independently as a verb, as illustrated in (32). Furthermore, it can be treated as a verb incorporating MOTION and PATH. This is evidenced by the fact that it can be affixed with the aspect marker -le.

$$
\begin{array}{clll}
\text { (32) John } & \text { guo } & \text { le } & \text { Yingjili Haixia } \\
\text { John } & \text { pass } & \text { ASP } & \text { English Channel } \\
\text { FIGURE } & \text { MOTION/PATH } & \text { GROUND }
\end{array}
$$

In contrast, the verb fei "to fly" cannot occur alone without guo in this context, as illustrated in (33).

$$
\begin{array}{clcl}
\text { (33) *John } & \text { fei } & \text { le } & \text { Yingjili Haixia } \\
\text { John } & \text { fly } & \text { ASP } & \text { English Channel } \\
\text { FIGURE } & \text { MOTION/MANNER } & \text { GROUND }
\end{array}
$$

The above illustrations also show that guo is a verb incorporating PATH and is the center of predication in the verb compound fei-guo, which indicates the completion of passing the channel. The sentence in (31) should be translated literally as in (34).

(34) John passed the English Channel by flying.

Similarly, the equivalent Chinese sentences for (28)-(30) can be given below in (35)-(37), which contain the resultative verb compounds piao-jin, piao-chu, and piao-guo. And, sentences in (38)-(40) show that the resultative complement V2 in each sentence is indeed a verb root incorporating PATH.

(35) pingzi piao-jin-le dongxue
bottle float-enter-ASP cave
"The bottle floated into the cave.”
(36) pingzi piao-chu-le dongxue
bottle float-exit-ASP cave
"The bottle floated out of the cave.”
(37) pingzi piao-guo-le yanshi
bottle float-pass(by)-ASP rock
"The bottle floated past the rock.”
(38) pingzi jin-le dongxue
bottle enter-ASP cave
"The bottle entered the cave.”
(39) pingzi chu-le dongxue
bottle exit-ASP cave-inside
"The bottle exited the cave.”
(40) pingzi guo-le yanshi
bottle pass-ASP rock
"The bottle passed by the rock.”

From the above illustrations, it appears that Chinese differs from both satellite-framed languages and verb-framed languages. In Talmy's typological classification of motion verbs, in addition to his tripartite classification, i.e., Motion + Path (verb-framed), Motion + Co-event (satellite-framed), and Motion + Figure, split system and intermixed system of conflation are also proposed. Based on a preliminary analysis, it appears that Chinese is neither a split system nor an intermixed system. 
In Chinese action-result verb compounds, V1 conflates Motion and Co-event, while V2 conflates Motion and Path. Thus Chinese presents a problem for Talmy's typological classification of motion verbs since it is both satellite-framed and verbframed depending whether we take V1 or V2 as the main verb. As the author has argued above, V2 is the center of predication of the action-result verb compounds, and therefore, can be treated as the main verb. If we take V2 as the main verb, then Chinese is no longer a satellite-framed language as Talmy has claimed. It makes more sense to view Chinese as primarily a verb-framed language and only secondarily a satellite-framed language. It would be interesting to see if other languages with action-result verb compounds show the same characteristics as Chinese.

\section{Conclusion}

The current thesis has shown that Chinese and English exhibit a systematic difference in structuring events consisting of both action and result. This systematic difference can be stated to the effect that while English structures on the action aspect, Chinese structures on the result aspect. In the spirit of linguistic relativism, I would like to view the difference as reflecting ontological relativity involving events. This ontological relativity means that English speakers tend to attend relatively more to the process of an event, but, in contrast, Chinese relatively more to the result. In other words, while English is an agentoriented language, Chinese is a patient-oriented language.

The ontological relativity suggested here is an extension of ontological relativity articulated by the philosopher Quine (1969). His well-known Gavagai example has illustrated two alternative ontological beliefs the speakers can hold about the referents of nouns. One is for nouns to refer to the "bodies" of objects, the other to the "materials" of objects. This difference has already been reflected in the semantic contrast between count and mass nouns in English. Yet, in terms of the linguistic relativity hypothesis, there is no distinction between count and mass nouns; that is, all nouns in classifier languages can be treated as mass nouns. Thus, in classifier languages, nouns are not inflected for plural and cannot be counted without the accompaniment of classifiers. In addition, bare nouns can indicate either definite or indefinite reference, depending on context. In other words, nouns in classifier languages denote materials or substances, non-discrete and unbounded, while in English and other European languages, they denote objects with discrete boundaries. This hypothesis, if it is to be tested for the cognitive consequences of linguistic relativity, would predict that native speakers of classifier languages would give prominence to material or substance, while native speakers of English and other European languages would give prominence to bodies.

Lucy (1992) designed an experiment to show that the mass noun hypothesis does have a cognitive consequence. The classifier language he used to contrast with English was Yucatec Maya. His subjects were ten Maya men and thirteen US Men. Subjects were presented with a triad of objects. Each triad consisted of an original object and alternative objects. The results show that English speakers overwhelmingly classify objects on the basis of shape, while Yucatec speakers overwhelmingly classify objects on the basis of materials. Cognitive differences induced by classifiers are further demonstrated in Zhang and Schmitt (1998). Their experiments showed that Chinese speakers, relative to English speakers, judged objects sharing a clas- sifier as more similar than objects not sharing a classifier and were more likely to recall them in clusters. From these two sets of experiments, it appears that language can affect certain types of cognition, if not thought as a whole. It is therefore worth exploring the question of whether the pervasive patterning of action-result schemas with the result as the center of information in Chinese can also affect certain aspects of cognitionor more specifically, conceptualization of reality, thus making us see the essence by the phenomenon.

The thesis lays great emphasis on how languages reflect people's different ways of conceptualizing the world and based on the study of Chinese and English verb-resultatives, it also attain some meaningful achievements. But the model described in the thesis only offers a very general guideline for similar studies. Since language and cognition involve so many elements and factors, there is a great unpredictability about the problems that may crop up. Nevertheless, one thing we can be sure of is that the problems are definitely more complicated and diverse than those that can be covered in one thesis. In addition, this research is done in a relatively small scale without exploring and analyzing relevant language materials exhaustively. If further research can be done in a larger scale with more materials included, it will be a big improvement on the current study. Besides, the similarity and difference between RVC constructions and resultative-de constructions are not discussed. Resultativede constructions are more analytic (in form) and more transparent (in meaning) than RVC constructions. Their comparison is a topic worthy of exploring.

\section{REFERENCES}

Bloom, A. H. (1981). The linguistic shaping of though: A study in the impact of language on thinking in China and the West. Hillsdale, NJ: Erlbaum.

Chang, J.-H. (2001). The syntax of event structure in Chinese. Honolulu: University of Hawaii Dissertation.

Chang, J.-H. (2003). Event structure and argument linking in Chinese. Language and Linguistics, 4, 317-351.

Chao, Y. R. (1968). A grammar of spoken Chinese. Berkeley and Los Angeles: University of California Press.

Cheng, L. L.-S., \& James Huang, C.-T. (1994). On the argument structure of resultative compounds. In $\mathrm{M}$. Chen, \& O. Tzeng (Ed.), Interdisciplinary studies on language and language change (pp. 187-221). Taipei: Pyramid.

Dowty, D. (1979). Word meaning and montague grammar. Dordrecht: Reidel Publishing Company. doi:10.1007/978-94-009-9473-7

Her, O.-S. (2007). Argument-function mismatches in mandarin resultatives: A lexical mapping account. Lingua, 117, 221-246. doi:10.1016/j.lingua.2006.01.002

Huang, C.-T. J. (1988). Wo pao de kuai and Chinese phrase structure. Language, 64, 274-311. doi:10.2307/415435

Huang, C.-T. J. (1992). Complex predicates in control. In R. Larson, U. Lahiri, S. Iatridou, \& J. Higginbotham (Eds.), Control and grammar (pp. 109-147). Dordrecht: Kluwer.

Lakoff, G. (1987). Women, fire, and dangerous things. Chicago: Chicago University Press. doi:10.7208/chicago/9780226471013.001.0001

Langacker, R. (1987). Foundations of cognitive grammar. Theoretical prerequisites. Stanford: Stanford University Press.

Langacker, R. (1991). Foundations of cognitive grammar. Descriptive application. Stanford: Stanford University Press.

Li, C. N., \& Sandra, A. T. (1981). Mandarin Chinese: A functional reference grammar. Berkeley: University of California Press.

Li, Y. F. (1999). Cross-componential causativity. Natural Language and Linguistic Theory, 17, 445-497. doi:10.1023/A:1006236828263

Lucy, J. (1992). Grammatical categories and cognition: A case study of 
the linguistic relativity hypothesis. Cambridge: Cambridge University Press. doi:10.1017/CBO9780511620713

Quine, W. (1969). Ontological relativity and other essays. New York: Columbia University Press.

Rapoport, T. R. (1993). Stage and adjunct predicates: Licensing and structure in secondary predication constructions. In E. Reuland, \& W. Abraham (Eds.), Knowledge and language volume II: Lexical and conceptual structure (pp. 157-182). Amsterdam: Kluwer Academic Publishers.

Rappaport, H., Malka, \& Beth, L. (2001). An event structure account of English resultatives. Language, 77, 766-797. doi:10.1353/lan.2001.0221

Simpson, J. (1983). Resultatives. In L. Levin, M. Rappaport, \& A. Zaenen (Eds.), Papers in lexical-functional grammar (pp. 143-158). Bloomington: Indiana University Linguistics Club.

Tai, J. H.-Y. (1999). Verb-copying in Chinese revisited. Chinese languages and linguistics 5: Interactions in language, 97-119. Taipei: Institute of Linguistics (Preparatory Office), Academia Sinica.

Talmy, L. (1985). Lexicalization patterns: Semantic structure in lexical forms. In T. Shopen (Ed.), Language typology and syntactic description (pp. 36-149). Cambridge: Cambridge University Press.

Talmy, L. (2000). Toward a cognitive semantic. Typology and process in concept structuring. Cambridge: MIT Press.

Thompson, \& Sandra, A. (1973). Resultative verb compounds in Mandarin Chinese: A case for lexical rules. Language, 49, 361-379. doi:10.2307/412459

Ungerer, F., \& Hans-Jorg, S. (1996). An introduction to cognitive lin- guistics. London: Longman.

Vendler, Z. (1967). Linguistics in philosophy. Ithaca: Cornell University Press.

Wu, C. H. F. (1994). A study of counterfactuals in Chinese and in English. Taipei: Crane Publishing Company.

Zhang, S., \& Bernd, S. (1998). Language-dependent classification: The mental representation of classifiers in cognition, memory, and ad evaluations. Journal of Experimental Psychology, 4, 375-385. doi:10.1037/1076-898X.4.4.375

Tai, J. (1989). Towards a cognition-based functional grammar of Chinese. Beijing: Beijing Language and Culture University Press.

Liu, C.-Z., \& Zhang, Y. (2012). On compound causatives and resultatives in Chinese. Journal of Changchun Normal University, 1, 70-74.

$\mathrm{Li}, \mathrm{F}$. Q. (2011). The mapping of composite thematic roles to grammatical functions in mandarin resultative verb compounds. Journal of Foreign Languages, 6, 19-25.

Shen, J.-X. (2011). Six lectures on Chinese grammar. Beijing: Commercial Press.

Shen, L. (1993). On resultative construction in Chinese. Linguistic Researches, 3, 12-21.

Wang, Q., \& Wu, Y.-Q. (2005). A comparative study of resultatives in Chinese and English. Journal of PLA University of Foreign Languages, 4, 17-23.

Wang, Y. (2008). The fourth paper on the new growth of linguistics: Exploration on post philosophy of language. Foreign Language Research, 4, 2-10. 